\begin{tabular}{|c|c|}
\hline Title & Lamellar-micelle transition in a hydrogel induced by polyethylene glycol grafting \\
\hline Author(s) & Haque, Md. A namul; Kurokawa, Takay uki; Gong, Jian Ping \\
\hline Citation & $\begin{array}{l}\text { Soft Matter, 9(21), 5223-5230 } \\
\text { https://doi.org/10.1039/c3sm50544h }\end{array}$ \\
\hline Issue Date & $2013-04$ \\
\hline DOC URL & http:/hdl.handle.net/2115/55284 \\
\hline Rights & Soft Matter, 2013,9, 5223-5230 - Reproduced by permission of The Royal Society of Chemistry (RSC) \\
\hline Type & article (author version) \\
\hline File Information & Haque_Lamellar Micelle transition.pdf \\
\hline
\end{tabular}

Instructions for use 


\section{Lamellar-Micelle Transition in Hydrogel Induced by Polyethylene Glycol Grafting}

Md. Anamul Haque, Takayuki Kurokawa, and Jian Ping Gong*

Faculty of Advanced Life Science, Hokkaido University, Sapporo 060-0810, Japan

*Tel. \& Fax: +81-11-706-2774, E-mail: gong@ sci.hokudai.ac.jp

*Corresponding Author

Keywords: liposome, bilayer membrane, polymer lipid, hydrogel, polymer grafting 


\begin{abstract}
We demonstrate, for the first time, a distinct change of the bulk properties of a hydrogel based on the lamellar-micelle transition. The hydrogel consists of thousands of periodical stacking of bilayer membranes, poly(dodecyl glyceryl itaconate) (PDGI), inside a dilute polyacrylamide network. Along with a bright structure color, the hydrogel exhibits strong anisotropy in its bulk properties. By grafting short chain polymer lipid, poly(ethylene glycol) dodecyl ether $\left(\mathrm{C}_{12} \mathrm{EO}_{23}\right)$, into the bilayer membrane, sharp changes in the structure color, swelling, and modulus of the hydrogel are observed in a narrow $\mathrm{C}_{12} \mathrm{EO}_{23}$ concentration range. These changes are ascribed to the structure transition of the single domain lamellar phase to the multi-domain micelle phase of PDGI, induced by the excluded volume effect of two dimensionally grafted poly(ethylene glycol) (PEG). By controlling the water permeable nanochannels formed during the structure transition, specific molecular diffusion and recognition are demonstrated. This lamellar bilayer hydrogel could be open a new insight into the lipid bilayer system to investigate the nature of biological membrane by the bulk behaviors of hydrogel.
\end{abstract}




\section{Introduction}

Liposomes are mainly composed of phospholipids such as phosphatidycholines, which form large single lamellar vesicles in the nanometer scale based on the bilayer self-assembly of phospholipids [1-3]. Sterically stabilized liposomes have been widely studied as a drug delivery system because of their unique nature of long circulation time in the blood stream [4-6]. The steric stabilization is done by liposome-bilayer component such as cholesterol [7], amphiphilic polyethylene glycol (PEG) covalently attached to a lipid [8]. PEG is an inert, water-soluble polymer which has been extensively used for intravenous liposome drug delivery because they can adapt different conformation $[9,10]$ and control the properties of membrane by modulating the membrane structure [11-13] by grafting on the bilayer membrane surface. For example, PEG grafted at the bilayer surface in the liposomes gives rise to steric interactions that stabilizes the lipid bilayer against the close approach of protein, cell membrane and thus increases the resistance of liposomes to leakage and degradation [1418]. Numerous attempts have been made to graft PEG in the bilayer surface for investigating the structure and nature of the bilayer membrane. However, most of the studies so far are limited to the nanometer scale $[2,3]$ because of the instability of liposome bilayer in macroscale.

Recently, we have successfully developed stable lamellar bilayer structure of macroscopic giant size in a soft, dilute polymer network of hydrogel system [19-21]. The sheet-like lamellar structure, of thousands bilayer membranes stacking in single domain, is formed from self-assembly of a lipid-like polymeric surfactant, poly(dodecyl glyceryl itaconate) (PDGI). The PDGI bilayers are stably entrapped in the chemically cross-linked polyacrylamide network by one-step individual polymerization of DGI and acrylamide (AAm) in the presence of a small amount of chemical cross-linker, to generate a stratified 
layer composite hydrogel, named as PDGI/PAAm [Figure 1a]. The thickness of polymerized DGI (PDGI) bilayer is $4.7 \mathrm{~nm}$ [22] and the center-to-center distance between two next neighboring PDGI bilayer membranes, $d$, is 150-300 $\mathrm{nm}$ depending on the DGI concentration and the swelling degree of PAAm networks. At room temperature, the single PDGI bilayer membrane is rigid with a modulus in the order of several MPa, and the PAAm matrix is soft with a modulus of few $\mathrm{kPa}[19,21]$. The PDGI/PAAm lamellar hydrogel exhibits visible color at various wavelength bands due to the Bragg's diffraction of visible light on the periodic lamellar membranes. The lamellar membranes are impermeable to water and other hydrophilic molecular species. Owing to the global uni-axial orientation of the lamellar bilayers, the water permeable PAAm network can swell freely in the direction perpendicular to the bilayers. However, the swelling of PAAm layer is completely constrained by the rigid, water-impermeable PDGI layers. As a result, the gel has a strong anisotropy in its bulk properties: it exhibits one-dimensional swelling in the direction perpendicular to the lamellar bilayers while the swelling in the direction of the bilayers is completely constrained. The modulus of the gel along the lamellar layer direction is c.a. 10 times higher than that in the vertical direction.

In this article, we intend to investigate how the lamellar bilayer structure entrapped in the hydrogel network changes with the perturbation of the other amphiphilic molecules with a bulky hydrophilic head group. How the bulk properties of the gel changes accompany with the possible structural changes of the bilayer. For this purpose, we employ a short chain polymer lipid, poly(ethylene glycol) dodecyl ether containing 23 ethylene oxide units $\left(\mathrm{C}_{12} \mathrm{EO}_{23}\right)$ (Figure 1b). The alkyl modified poly(ethylene glycol) (simply PEG) chain can be grafted into the bilayer membranes through hydrophobic association. 
The properties of the PDGI/PAAm gel, such as structure color, swelling, and modulus, dramatically change in a narrow PEG grafting concentration, indicating the structure transition from bilayer phase to micellar phase. This structure transition leads to nanochannel formation in the bilayer membrane of the hydrogel. The nano-channel created in the bilayer membrane by PEG grafting demonstrates the potential use of this hydrogel as drug delivery system where controlled release of particular drug molecule is required. This lamellar bilayer hydrogel system could be an ideal model to investigate the liposome system in the field of liposome-based drug delivery system. To the best of our knowledge, this is the first work to relate the nature of nanosacle lamellar membrane, lamellar-micelle transition, to the bulk behaviors of a hydrogel. 


\section{Experimental}

\subsection{PDGI/PAAm gel preparation}

The parent PDGI/PAAm gel, which shows yellowish-green color (diffraction peak wavelength $\lambda_{\max } \sim 550 \pm 5 \mathrm{~nm}$ measured at a Bragg's angle of $60^{\circ}$ ), was used for the PEG grafting study. The gel was prepared by simultaneous free radical polymerization from aqueous solution of $0.10 \mathrm{M}$ dodecyl glyceryl itaconate [DGI: $n$ $\left.\mathrm{C}_{12} \mathrm{H}_{25} \mathrm{OCOCH}_{2} \mathrm{C}\left(=\mathrm{CH}_{2}\right) \mathrm{COOCH}_{2} \mathrm{CH}(\mathrm{OH}) \mathrm{CH}_{2} \mathrm{OH}\right], 0.025$ mol\% sodium dodecyl sulfate (SDS) of DGI, $2 \mathrm{M}$ acrylamide (AAm), $2 \mathrm{mM} N, N^{\prime}$-methylenebis (acrylamide) (MBAA) as a cross-linker of AAm, and $2 \mathrm{mM}$ Irgacure as an initiator. The detailed procedures were described in our previous paper [19]. Briefly, the precursor solution was prepared at $55^{\circ} \mathrm{C}$ for about $4 \mathrm{~h}$ in water bath until the equilibrium formation of stable micro-domain lamellar bilayers of self-assembled DGI. Prior to the polymerization, bilayer micro-domains were aligned in one direction in the macroscopic scale by applying a shear flow to the precursor solution. For this purpose, a sheet-like polymerization cell $\left(10 \times 1 \times 0.05 \mathrm{~cm}^{3}\right)$ was prepared by sandwiching silicone rubber between two glass plates. Then the precursor solution was injected into the reaction cell with a high flow speed of about $5 \mathrm{cms}^{-1}$ which created a strong shear rate of about $200 \mathrm{~s}^{-1}$. Due to the strong shear flow between two parallel glass plates, thousands of bilayers (cm-scale) were aligned in one direction parallel to the substrate surfaces. After polymerization, bilayers were stably entrapped in the PAAm matrix to give a mechanically strong hydrogel after attaining equilibrium swelling in water. A temperature of $50-55^{\circ} \mathrm{C}$ was maintained during injection and polymerization process to prevent DGIlamellar phase separation. The PAAm gel was prepared similarly in the absence of DGI and SDS. 


\subsection{PEG grafting}

The grafting of the bilayer was simply performed by immersing the water swollen PDGI/PAAm gel into aqueous solution of an amphiphilic short chain polymer lipid: poly(ethylene glycol) dodecyl ether containing 23 ethylene oxide units $\left(\mathrm{C}_{12} \mathrm{EO}_{23}\right)$ of different concentrations for 2-3 weeks until attaining the equilibrium state. This gel was cut into small pieces $\left(\sim 4 \times 1 \times 0.12 \mathrm{~cm}^{3}\right)$ to shorten the equilibrium time of grafting of the poly(ethylene glycol) (PEG) chain into the lamellar membrane of the gel. After grafting the gels were further washed with water for several times in 2-3 weeks. The concentration of $\mathrm{C}_{12} \mathrm{EO}_{23}$, i.e., PEG is expressed in terms of mole ratio of $\mathrm{C}_{12} \mathrm{EO}_{23}$ to DGI, $\mathrm{n}_{\mathrm{PEG}} / \mathrm{n}_{\mathrm{DGI}}$.

\subsection{Reflection Spectrum}

A Xe lamp was used as light source to obtain the reflection spectrum. Reflection measurement optics of variable angle (Hamamatsu Photonics KK, C10027A10687) was used to detect the reflected light. A photonic multichannel analyzer (Hamamatsu Photonics KK, C10027) was used for analyzing the detected signal. The entire reflection spectrum was obtained by keeping both the incident (Bragg's angle) and reflection angles at $60^{\circ}$, and the wavelength at maximum, $\lambda_{\max }$, was obtained from the reflection spectrum. The distance between two lamellar layers, $d$, was determined using the Bragg's law of diffraction, $2 n d \sin \theta$ $=\lambda$, where $n=1.33$ (refractive index of water), $\theta$ is the Bragg angle or incident angle, and $\lambda$ is the wavelength at maximum of the reflection spectrum [23].

\subsection{Swelling ratio measurement}

As revealed by the previous paper, the in-plane structure of the bilayer membranes of the PDGI/PAAm gel is isotropic [19]. So the swelling ratios of the gel parallel to the bilayer membranes, so-called as the swelling ratio along the length $\left(L_{1} / L_{0}, L_{2} / L_{1}\right)$ in this paper, was 
obtained by measuring the length $L$ of the sheet shaped gel. The swelling ratios perpendicular to the bilayer membranes $\left(T_{1} / T_{0}, T_{2} / T_{1}\right)$ was obtained by measuring the thickness $(T)$ of the gel. Here the subscript " 0 ", "1", and "2" represent the corresponding values in the asprepared state, equilibrium state in pure water, and equilibrium state in $\mathrm{C}_{12} \mathrm{EO}_{23}$ solution, respectively. The length of the gel at different swollen state was measured by a slide calipers and the thickness was measured by a tensile-compressive test machine (Tensilon RTC-1310A, Orientec Co.).

\subsection{Mechanical testing and modulus measurement}

The gels were cut into dumbbell shape of standardized size by the gel cutter (JIS- 6251-7) and the tensile stress-strain measurement was performed along the direction parallel to the bilayer membranes using a commercial test machine (Tensilon RTC-1310A, Orientec Co.). The elongation velocity was maintained as $200 \mathrm{~mm} / \mathrm{min}$. The elastic modulus parallel to the bilayer membranes, defined as $E=\Delta \sigma / \Delta \varepsilon$, was calculated from initial slope of the linear elastic loading portion (10\% elongation of the initial gauge length of gel sample) of the tensile stress-strain curves. Here, $\sigma$ is the nominal stress defined as the force per unit area of initial cross-section of the gel sample and $\varepsilon$ is the strain defined as $\varepsilon=\left(l-l_{0}\right) / l_{0}$ where $l_{0}$ is the initial gauge length of the gel sample and $l$ is its current length during elongation.

\subsection{Polarizing optical microscope (POM) observations}

The structure anisotropy of the gel was observed by the POM (Nikon Eclipse LV100POL) under crossed Nicol at room temperature. The gel was also observed under parallel polarizers. The observation was performed from the cross section (side view) of the sheet-like gels that were placed on a glass substrate. 


\section{Results and discussion}

The photographs of the lamellar hydrogel before and after immersing in poly(ethylene glycol) dodecyl ether, $\mathrm{C}_{12} \mathrm{EO}_{23}$, (abbreviated hereafter as PEG) solutions are shown in Figure 2a. At low PEG concentration $\left(n_{\mathrm{PEG}} / n_{\mathrm{DGI}}=1 / 40\right)$, the color of the gel hardly changes. In contrast, the color of the gel largely shifts to blue-violet at high PEG concentration (1/17 and 1/5). The corresponding reflection spectra of the gel swelled in PEG solutions of various mole ratios are shown in Figure $\mathbf{2 b}$. The moderately sharp spectra at high PEG concentrations indicate that the periodically ordered lamellar structure is retained even after PEG grafting. The birefringence observed by the polarizing optical microscope at crossed nicol for the cross sectional view of the plate-like gel at high PEG concentration $\left(n_{\mathrm{PEG}}: n_{\mathrm{DGI}}=1: 1\right)$ also justifies this (Figure 2c). The complete dark image is observed when the sample located along the polarizer (Figure 2c, left) whereas bright image is produced when the sample aligned at an angle of $45^{\circ}$ (Figure 2c, right). This indicates that the bilayer structure in gel keeps its uniaxial orientation similar to the parent PDGI/PAAm gel [19] even after soaked with high PEG concentration. The swelling ratio at as prepared, water swollen, and PEG swollen states are demonstrated in Figure 2d. After immersing in water, the as prepared PDGI/PAAm gel shows an increase in swelling ratio perpendicular to the lamellae $\left(T_{1} / T_{0}=2.4\right)$ whereas no swelling observed at all parallel to lamellae $\left(L_{1} / L_{0}=1\right)$. After soaking with high concentration of PEG $\left(n_{\mathrm{PEG}}: n_{\mathrm{DGI}}=1: 1\right)$, the PDGI/PAAm gel exhibits an increase and decrease in swelling ratio, respectively, parallel $\left(L_{2} / L_{1} \sim 1.43\right)$ and perpendicular $\left(T_{2} / T_{1} \sim\right.$ 0.68) to the bilayer. The contrast swelling in PEG solution results the gel to reach an isotropic swelling state of the single PAAm gel (swelling ratio 1.5), since the net swelling ratio of the PDGI/PAAm gel after soaked with PEG reaches to almost identical value $\left(L_{2} / L_{0} \sim\right.$ $\left.1.43, T_{2} / T_{0} \sim 1.55\right)$. This indicates a sharp transition of the gel's property with the grafting of PEG. 
The diffraction peak wavelength $\left(\lambda_{\max }\right)$, gel dimension, modulus of bulk gel and bilayer, before and after PEG grafting over a wide concentration range are shown in Figure 3a. A sharp decrease in $\lambda_{\max }$ is observed at $\mathrm{n}_{\mathrm{PEG}} / \mathrm{n}_{\mathrm{DGI}} \sim 1 / 25$ until saturation at a concentration of $n_{\mathrm{PEG}} / \mathrm{n}_{\mathrm{DGI}} \sim 1 / 20$, whereas below and above this concentration range, $\lambda_{\max }$ does not change significantly. We assume that the blue shift in color of gel is emerged due to the decrease in inter bilayer distance $(d)$. To confirm this, we characterize the swelling behaviors of the gel in terms of relative size change parallel and perpendicular to bilayers of the gel after being immersed in various PEG solutions (Figure 3b). In agreement with the sharp change of $\lambda_{\max }$ with PEG concentration, the thickness ratio of gel in PEG solution and in water $\left(T_{2} / T_{1}\right)$, i.e., swelling ratio perpendicular to the bilayers of the gel, decreases in the same intermediate PEG concentration range $\left(n_{\mathrm{PEG}} / \mathrm{n}_{\mathrm{DGI}}=1 / 25\right.$ to $\left.1 / 10\right)$. Here the subscripts 0,1 , and 2 stand for the as-prepared state, water-swollen state, and PEG grafting state, respectively. Below and above this concentration range, the value of $T_{2} / T_{1}$ remains almost constant. Furthermore, we observe that $d / d_{0}=T_{2} / T_{1} \sim 0.7$, where $d$ is the inter-bilayer distance estimated from the $\lambda_{\max }$. This observation justifies that the blue shift is originated from the decrease in the thickness of the PAAm layers $(150-250 \mathrm{~nm})$. On the other hand, the swelling ratio parallel to the bilayer $L_{2} / L_{1}$ is in opposite to the behavior of $T_{2} / T_{1}$. It remains unchanged $\left(L_{2} / L_{1}=1\right)$ at low PEG concentration but increases abruptly in the intermediate PEG concentration range $\left(\mathrm{n}_{\mathrm{PEG}} / \mathrm{n}_{\mathrm{DGI}}\right.$ $=1 / 25$ to $1 / 10)$, and finally reaches to a constant value $\left(L_{2} / L_{1} \sim 1.4\right)$ above the PEG concentration of $n_{\mathrm{PEG}} / \mathrm{n}_{\mathrm{DGI}}=1 / 10$. We have observed that the swelling ratio of the PAAm gel in the various PEG solution does not change over the whole concentration range of PEG investigated, indicating that there is no distinct interaction between PEG and the PAAm polymer network. Therefore, the lateral expansion of the gel is caused by the lateral 
expansion of bilayers upon the grafting of PEG. During this expansion process, the continuous bilayer should be destroyed to form discontinuous micelle phase.

As shown in Fig 3a, rather than an increase, the inter-bilayer distance decreases from $250 \mathrm{~nm}$ to $150 \mathrm{~nm}$ after PEG grafting. This excludes any possible inter-layer repulsion through the grafted PEG chains. In fact, the size of grafted PEG chains (contour length $\sim 2$ $\mathrm{nm}$ ) is much smaller than the inter-layer distance. We assume that the swelling of the bilayers along the bilayer direction accounts for the decrease in the inter bilayer distance due to the volume constraint effect. It should mention that the overall volume of the gel almost keeps constant over the whole PEG concentration range investigated. When the as-prepared PDGI/PAAm gel is immersed in water, the PAAm matrix layers can only swell $\left(T_{1} / T_{0}=2.4\right)$ in the thickness direction, i.e., perpendicular to the bilayers, whereas the swelling of PAAm along the length axis, i.e., parallel to the bilayers, is completely constrained by the nonswellable PDGI bilayers $\left(L_{1} / L_{0}=1.0\right)$ [19]. That is, the swelling of the PAAm soft layers is anisotropic in water. Once the bilayer packing is destroyed by the PEG grafting, the anisotropic swelling of the soft PAAm layers is relaxed partially to isotropic swelling. As a result, the inter-bilayer distance, $d$, decreases in compensate to the increase in the lateral swelling.

Concomitant with the lateral swelling of the gel $\left(L_{2} / L_{0} \sim 1.4\right)$, a sharp decrease in the elastic modulus of the bulk gel $\left(E_{\mathrm{PDG} / \mathrm{PAAm}}\right)$ is also observed in the same concentration range $\left(\mathrm{n}_{\mathrm{PEG}} / \mathrm{n}_{\mathrm{DGI}}=1 / 25\right.$ to $\left.1 / 10\right)$. The $E_{\mathrm{PDG} / \mathrm{PAAm}}$ saturates to $\sim 20 \mathrm{kPa}$ at the high PEG concentration, of the similar level of PAAm $\left(E_{\mathrm{PAAm}} \sim 8 \mathrm{kPa}\right)$, as shown in Figure 3c. This dramatic decrease in the modulus of the lamellar gel also justifies the structure change, from the continuous lamellar bilayer membrane phase (macro-domain) to discontinuous phase (micro- or nanodomain). 
As the PDGI/PAAm hydrogel can be considered as a composite consisting of alternating rigid layers of bilayer membrane and soft layers of PAAm network, we can calculate the elastic modulus of the single bilayer membrane using the layer structure model $[24,25]$

$$
E_{\|}=\alpha E_{\text {bilayer }}+(1-\alpha) E_{\mathrm{PAAm}}
$$

where, $E_{\|}, E_{\text {bilayer }}$, and $E_{\mathrm{PAAm}}$ are the elastic moduli of the bulk PDGI/PAAm gel parallel to the bilayers, the single PDGI bilayer membrane, either in continuous state or discontinuous state, and the single PAAm network layer, respectively. $\alpha$ is the thickness fraction of the PDGI bilayer and 1- $\alpha$ is that of the PAAm network layer. Accordingly, $\alpha=d_{\text {bilayer }} / d$, where $d_{\text {bilayer }}(=4.7 \mathrm{~nm})$ is the thickness of one PDGI bilayer and $d$ is the inter-planer distance between two next neighboring bilayers. Using Equation 1, the elastic modulus of a single bilayer membrane ( $E_{\text {bilayer }}$ ) upon stretching is calculated from the bulk tensile modulus of the gel $\left(E_{\|}\right)$and the $\lambda_{\max }$ of the reflection spectrum. The $E_{\text {bilayer }}$ are also plotted as a function of PEG concentration (Figure 3c). The modulus of one single bilayer dramatically changes from 6.0 MPa to 0.14 MPa which justify formation of discontinuous membranes possibly micellar domains from a continuous bilayer phase after PEG grafting. This result indicates that after structure transition, the discontinuous bilayer membranes and/or micellar domains are connected by soft and water permeable PAAm phases due to the grafting of PEG.

As have been revealed in our previous paper, the stratified lamellar bilayers not only diffract light to exhibit magnificent structural color but also serve as reversible sacrificial bonds to dissipate energy and toughen the hydrogel dramatically [20,21]. The structure transition of the bilayers from continuous phase to discontinuous phase should also substantially influence the mechanical strength of the hydrogel. Figure 4 shows the tensile elongation curves of the PDGI/PAAm, PEG grafted PDGI/PAAm gel, and the PAAm gel. A 
clear yielding appears at an elongation ratio of $\sim 1.5$ for PDGI/PAAm gel whereas no yielding appears for the PEG grafted PDGI/PAAm gel. The latter showed almost similar deformation behavior with PAAm gel. The yielding reveals the bilayer structure change, i.e., dissociation of hydrophobically aggregated PDGI bilayers by elongation [20]. Disappearance of yielding for the PEG grafted gel indicates the absence of significant change in bilayer structure on elongation because the bilayers already experienced an elongation of 1.4 times by PEG grafting. The dilation of the bilayer membrane causes the structure transition of the membrane, either by elongation of external stress, a uniaxial event, or by PEG-induced swelling, a biaxial event. It is interesting to observe that the strain-induced and the PEG grafting induced structure change occur at the similar dilation extend (elongation ratio 1.5, swelling ratio $\left.L_{2} / L_{1} \sim 1.4\right)$. The strength of the gel substantially decreases due to preelongation $\left(L_{2} / L_{1} \sim 1.4\right)$ by PEG grafting.

Next, we discuss what determine the critical PEG concentration to cause the structure transition of the bilayer membrane. Assuming that the project area of one grafted PEG on the bilayer membrane is $A_{\mathrm{PEG}}$ and the area of one head group of DGI unit is $A_{\mathrm{DGI}}$. The ratio $A_{\mathrm{DG}} /$ $A_{\text {PEG }}$ corresponds to the number of DGI units occupied by one PEG chain. Therefore, the critical mole ratio $\mathrm{n}_{\mathrm{PEG}} / \mathrm{n}_{\mathrm{DGI}}$ that the grafted PEG chains start to overlap is given by $\Phi^{*}=A_{\mathrm{DGI}} /$ $A_{\mathrm{PEG}}$. So the PEG concentration can also be expressed in relative to the critical mole ratio $\Phi^{*}$ of overlapping,

$$
\Phi / \Phi^{*}=\left(\mathrm{n}_{\mathrm{PEG}} / \mathrm{n}_{\mathrm{DGI}}\right) /\left(A_{\mathrm{DGI}} / A_{\mathrm{PEG}}\right)=\mathrm{n}_{\mathrm{PEG}} A_{\mathrm{PEG}} / \mathrm{n}_{\mathrm{DGI}} A_{\mathrm{DGI}}
$$

The area occupied by the head group of DGI unit on the bilayer surface is $A_{\mathrm{DGI}}=0.264 \mathrm{~nm}^{2}$ [22]. On the other hand, the size of the grafted PEG chain $A_{\mathrm{PEG}}$ can be estimated from the scaling theory. As the PEG chain is shorter than the size to exhibit significant excluded- 
volume interactions within the chain, we can adopt a random-walk conformation, which gives the radius of gyration of the chain $R_{\mathrm{g}}[26]$.

$\left\langle R_{\mathrm{g}}\right\rangle=a N^{1 / 2}$

Here, $a$ and $N$ are the monomer length and the number of monomer segments of PEG, respectively. $A_{\mathrm{PEG}}=\pi\left\langle R_{\mathrm{g}}{ }^{2}\right\rangle=6.5 \mathrm{~nm}^{2}$; where $a=0.3 \mathrm{~nm}$ and $N=23$.

We replot the PEG concentration in Figure 3 in terms of $\Phi / \Phi^{*}$, as shown by the upper horizontal axis of the figure. We observed that the structure transition occurs at $\Phi / \Phi^{*}=1$, which is in well agreement with the theoretically estimated overlap concentration of the PEG chains. This excellent agreement between the observation and the estimation demonstrates that PEG chains are homogeneously grafted on the bilayer membrane surfaces of the gel.

When $\Phi / \Phi^{*}<1$, there is no interaction between the grafted PEG chains and the polymer chains take mushroom-like conformation that is the most thermodynamically stable state (Figure 5a). Therefore, the swelling ratio, modulus, and structure color do not affect by the PEG grafting in this concentration range. When the PEG grafting concentration increases to a critical value of $\Phi / \Phi^{*} \sim 1$, the steric repulsive force exerts laterally to avoid overlapping between PEG chains (Figure 5b). Further increase in the grafting density will cause the change of PEG chain conformation from random coil (mushroom) state to extended (brush) state, which requires a huge decrease in entropy as penalty. When $\Phi / \Phi^{*}>1$, the steric repulsive energy exceeds the energy gain of hydrophobic association, the bilayer membrane initially tends to expand laterally followed by breaking into discontinuous phases, as justified by the change in swelling ratio, modulus, and structure color. After saturation, we assume that the PEG grafting density in the bilayers remains constant. On the other hand, the bulky hydrophilic head group (PEG chain) of the polymer lipid favors the formation of micelles. 
Because, when PEG forms brush regime at high grafting density the effective packing parameter become much smaller than $1 / 3$ [3]. Therefore, the lateral expansion of bilayers and the favorable micellization of the polymer lipids lead to a perturbation of the lipid bilayer membrane surfaces at higher grafting densities. At this stage, a phase transition, is supposed to be occurred and the macro-domain lamellar membrane splits into small domain bilayer membrane or micellar domains (Figure 5c). Evidence for such lateral expansion is provided by the increase in motional freedom of lipid bilayers on incorporating polymer grafted lipids in bilayer membranes and a transitions of bilayer into micellar phase as a result of polymer grafting are available in literature [9,27,28].

Next, we discuss the size of micelle domains. If we assume that the bilayer splits into small domains of an average radius $R$ and the distance between next neighbouring domains is determined by the PEG size $2 R_{\mathrm{g}}$, then $\left(2 R+2 R_{\mathrm{g}}\right) / 2 R=L_{2} / L_{1}$. As $L_{2} / L_{1}=1.4$, we have $R=5 \mathrm{~nm}$, where $R_{\mathrm{g}} \sim 2 \mathrm{~nm}$ for PEG brush regime. This indicates that after transition, the lamellar bilayers split into micelle domains of average diameter $2 R \sim 10 \mathrm{~nm}$, which is close to liposome [3]. The channel size between the domains is $2 R_{\mathrm{g}} \sim 4 \mathrm{~nm}$, as illustrated in Figure 5c.

The formation of nano-channel in the bilayer membrane was further confirmed by observing the diffusion of a dye (Allura red) into both grafted and non-grafted PDGI/PAAm gels (Figure 6). The images in the left two columns are for both PDGI/PAAm gel and grafted PDGI/PAAm gels taken with black and white background. After immersing the gels in dye solution for 5 min (images in $3^{\text {rd }}$ column), both of them show red color. After washing with water, the grafted gel still remains red color in the whole sample, however, the non-grafted gel show red only in the edge of the gel whereas the middle part are still the same as that before dying (images in $4^{\text {th }}$ column). If we compare the images for grafted and non-grafted gels which taken at black background, one can see easily that the grafted gel changes its color 
completely from green to blue but the non-grafted gel changes its color from green to blue only in the edge. Therefore, dye solution can diffuse in the grafted gel parallel as well as perpendicular to the PDGI bilayers which is in contrast to non-grafted gel where dye can only diffuse from the side but completely restricted through the impermeable lamellar membrane. This indicated clearly that the membrane channel formed in the grafted gel.

\section{Conclusions}

In conclusion, we have successfully grafted poly(ethylene glycol) (PEG) chains to the lamellar bilayer of the anisotropic PDGI/PAAm hydrogel using a polymer lipid, poly(ethylene glycol) dodecyl ether $\left(\mathrm{C}_{12} \mathrm{EO}_{23}\right)$. By the grafting of PEG, the bilayer membrane undergoes a structure transition from a lamellar phase to a micellar phase around the overlapping concentration of PEG chains at bilayer surface. In accompany to the structure change, the gel exhibits dramatic changes in visible light diffraction, swelling, modulus, and solute diffusion anisotropy of the bulk hydrogel. The uni-domain bilayer membrane exhibits a very high modulus of about $6 \mathrm{MPa}$, whereas after transition to micro-domain micelles, the PDGI bilayer shows a modulus of $0.1 \mathrm{MPa}$. The results that we obtained in this study are found in well agreement with the theoretical model for the PEG grafting on membrane surface. The nano-channels created in the membrane significantly increase the permeability of the membrane which can transport specific molecules as the smart drug delivery system. Moreover, the PDGI/PAAm hydrogel is an excellent system to investigate and characterize more deeply the nature of biological membrane upon polymer grafting that could correlate the behavior of nanoscale membrane with the macroscale system.

\section{ACKNOWLEDGEMENTS}


This study was supported by a Grant-in-Aid for Specially Promoted Research (No. 18002002) from the Ministry of Education, Science, Sports and Culture of Japan. 


\section{References}

1 O.G. Mouristen and K. Jorgenson, Chem. Phys. Lipids, 1994, 73, 3-25.

2 Z. Varga, A. Wacha, U. Vainio, J. Gummel and A. Bota, Chem. Phys. Lipids, 2012, 165, 387- 392 .

3 O. Garbuzenko, Y. Barenholz and A. Priev, Chem. Phys. Lipids, 2005, 135, 117-129

4 Y. Barenholz. Curr. Opin. Colloid Interface Sci. 2001, 6, 66-77.

5 D. D. Lasic and F. Martin, Stealth Liposomes, CRC Press, Boca Raton, FL, 1995.

6 D. D. Lasic, and D. Papahadjopoulous, Medical Applications of Liposomes, Elsevier Science, Amsterdam, 1998.

7 A. M. Samuni, A. Lipman, and Y. Barenholz, Chem. Phys. Lipids, 2000, 105, 121134.

8 S. Zalipsky, and M. J. Harris, Introduction to chemistry and biological applications of poly(ethylene glycol), In: J.M. Harris and S. Zalipsky (Eds.), Poly(ethylene glycol), Chemistry and Biological Applications. ACS Symposium Series 680. American Chemical Society, Washington, DC, 1997.

9 D. Marsh, R. Bartucci and L. Sportelli, Biochim. Biophys. Acta, 2003, 1615, 33- 59.

10 D. Needham and D. H. Kim, Colloids and Surfaces B: Biointerfaces, 2000, 18, 183195.

11 T. H. Epps, D. M. DeLongchamp, M. J. Fasolka, D. A. Fischer and E. L. Jablonski, Langmuir, 2007, 23, 3355-3362. 
12 U. Raviv, S. Giasson, N. Kampf, J. F. Gohy, R. Jerome and J. Klein, Nature, 2003, 425, 163-165.

13 H. B. Zeng, Y. Tian, B. X. Zhao, M. Tirrell and J. Israelachvili, Langmuir, 2009, 25, 4954-4964.

14 F. J. Xu, K. G. Neoh and E. T. Kang, Prog. Polym. Sci., 2009, 34, 719-761.

15 T. Masui, M. Imai, K. Nakaya and T. Taniguchi, J. Chem. Phys. 2006, 124, 074904.

16 D. D. Lasic, F. Martin, A. Gabizon, S. Huang, and D. Papahadjopoulos, Biochim. Biophys. Acta 1991, 1070, 187.

17 J. M. Harris, Ed. Poly(Ethylene Glycol) Chemistry: Biotechnical and Biomedical Applications; Plenum Press: New York, 1992.

18 J. M. Harris, S. Zalipsky, Eds. Poly(Ethylene Glycol) Chemistry and Biological Applications; American Chemical Society: Washington, DC, 1997.

19 M. A. Haque, G. Kamita, T. Kurokawa, K. Tsujii and J. P. Gong, Adv. Mater., 2010, 22,5110 .

20 M. A. Haque, T. Kurokawa, J. P. Gong, Soft Matter, 2012, 8, 8008.

21 M. A. Haque, T. Kurokawa, G. Kamita and J. P. Gong, Macromolecules, 2011, 44, 8916.

22 K. Naitoh, Y. Ishii and K. Tsujii, J. Phys. Chem. 1991, 95, 7915.

23 M. A. Haque, T. Kurokawa, G. Kamita, Y. Yue and J. P. Gong, Chem Mater, 2011, 23, 5200 . 
24 K. Okumura and P. G. de-Gennes, Eur. Phys. J. E., 2001, 4, 121.

25 A. Nakayama, A. Kakugo, J. P. Gong, Y. Osada, M. Takai, T. Erata, and S. Kawano, Adv. Funct. Mater., 2004, 14, 1124.

26 A. Dittmore, D. B. McIntosh, S. Halliday and O. A. Saleh, Phys. Rev. Lett., 2011, 107, 148301.

27 G. Montesano, R. Bartucci, S. Belsito, D. Marsh, and L. Sportelli. Biophys. J., 2001, 80, $1372-1383$.

28 K. Hristova and D. Needham, Macromolecules, 1995, 28, 991-1002. 
(a)

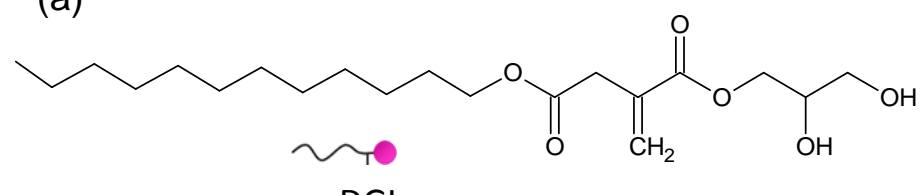

DGI
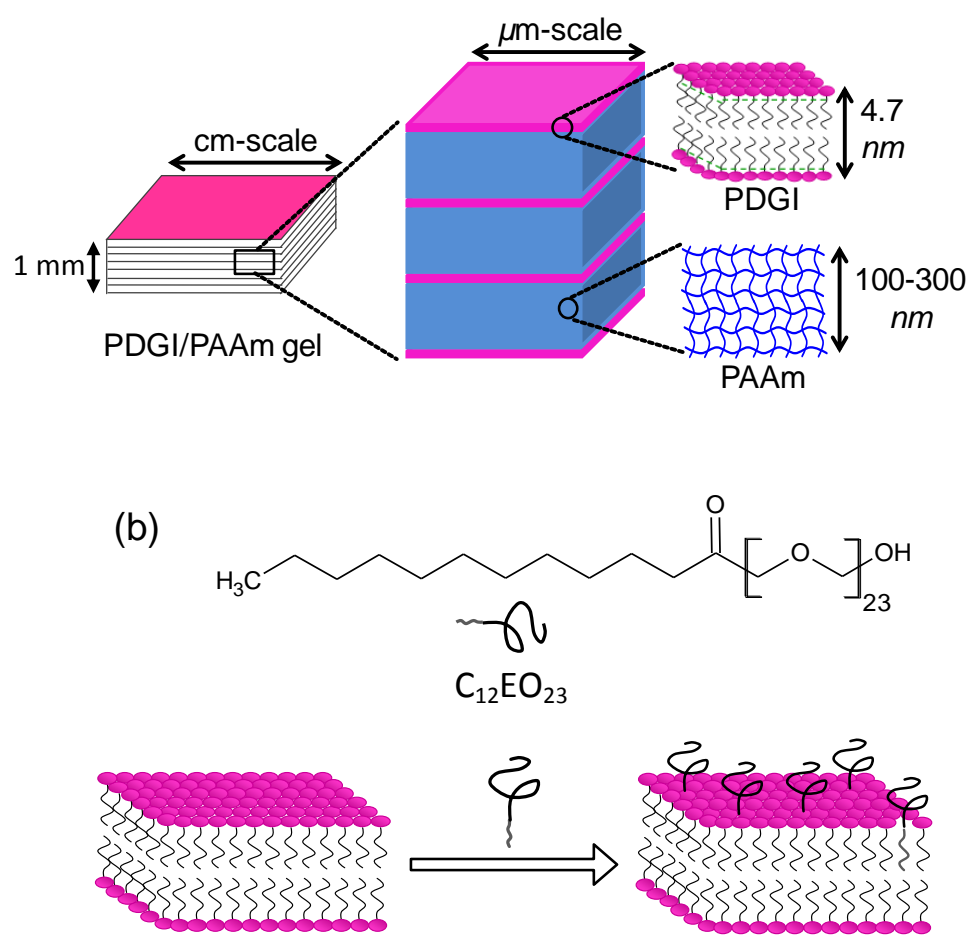

Figure 1: (a) A 3D illustration of the PDGI/PAAm gel and the molecular structure of DGI (dodecyl glyceryl itaconate). The gel consists of several thousands of soft PAAm and hard PDGI layers. (b) Schematic representation of a poly(ethylene glycol) dodecyl ether $\left(\mathrm{C}_{12} \mathrm{EO}_{23}\right)$, and the incorporation of the alkyl modified poly(ethylene glycol) (PEG) through its alkyl chain into self-assembly bilayer structure of DGI in the PDGI/PAAm hydrogel. 
(a)

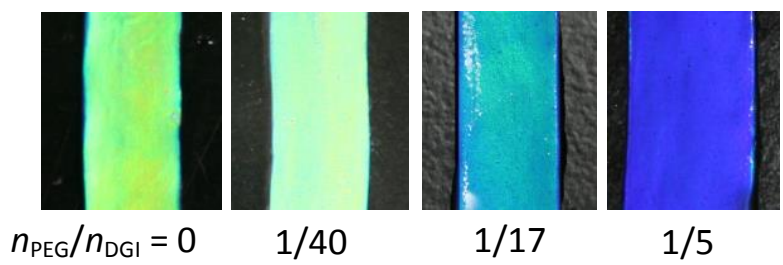

(b)

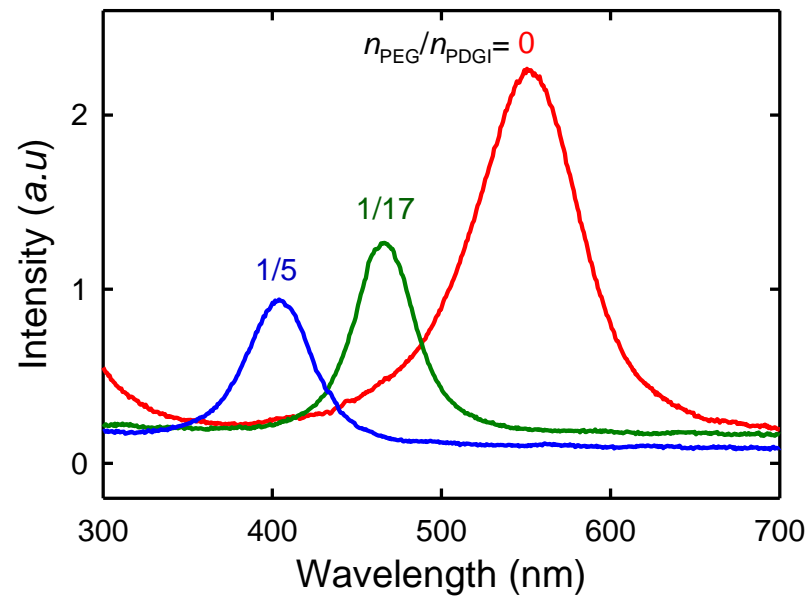

(c)
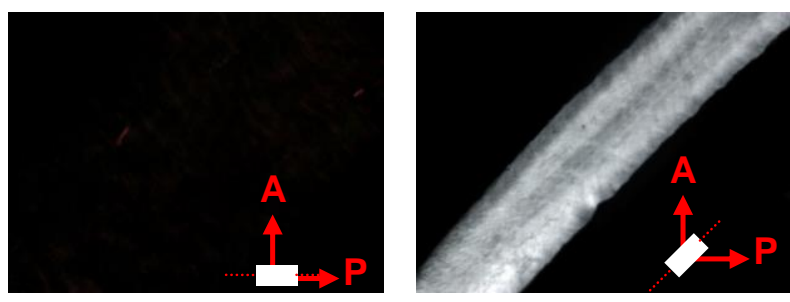

(d)

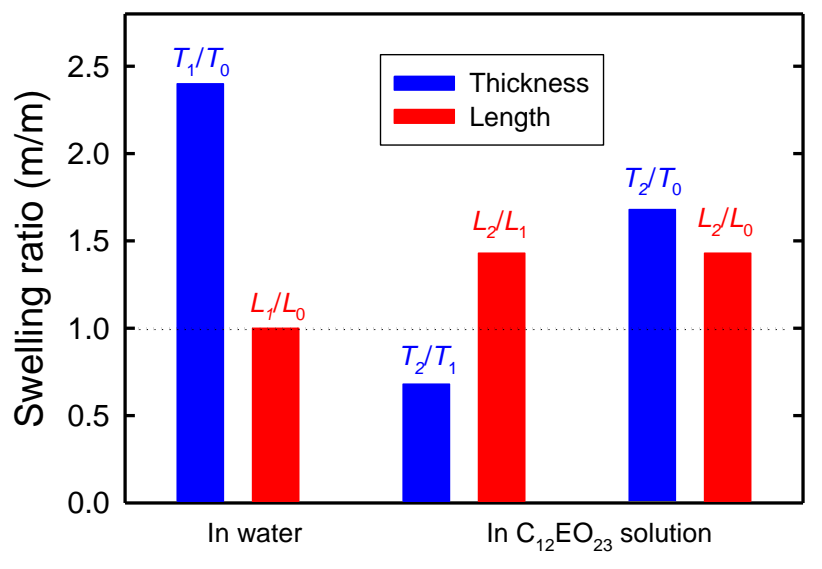

Figure 2: (a) Photographs of the PDGI/PAAm gel swollen at different mole ratios of PEG with DGI. (b) Reflection spectra shows the shift of peak position from higher to lower wavelength in accordance with the blue shift of gel color with increase of PEG ratio. (c) Polarizing optical microscope images taken under crossed nicol for the cross sectional view of the plate-like gel at high PEG concentration $\left(n_{\mathrm{PEG}}: n_{\mathrm{DGI}}=1: 1\right)(\mathrm{A}:$ Analyzer; P: Polarizer). (d) Swelling ratio of the PDGI/PAAm gel soaked with $\left(n_{\mathrm{PEG}}: n_{\mathrm{DGI}}=1: 1\right)$ and without PEG solution $\left(n_{\mathrm{PEG}}=0\right)$ normalized to the as prepared $\left(L_{1} / L_{0}, T_{1} / T_{0}, L_{2} / L_{0}, T_{2} / T_{0}\right)$ and water swollen $\left(L_{2} / L_{1}, T_{2} / T_{1}\right)$ PDGI/PAAm gel. Here the subscripts 0,1 , and 2 stand for the asprepared state, water-swollen state, and PEG grafting state, respectively. 


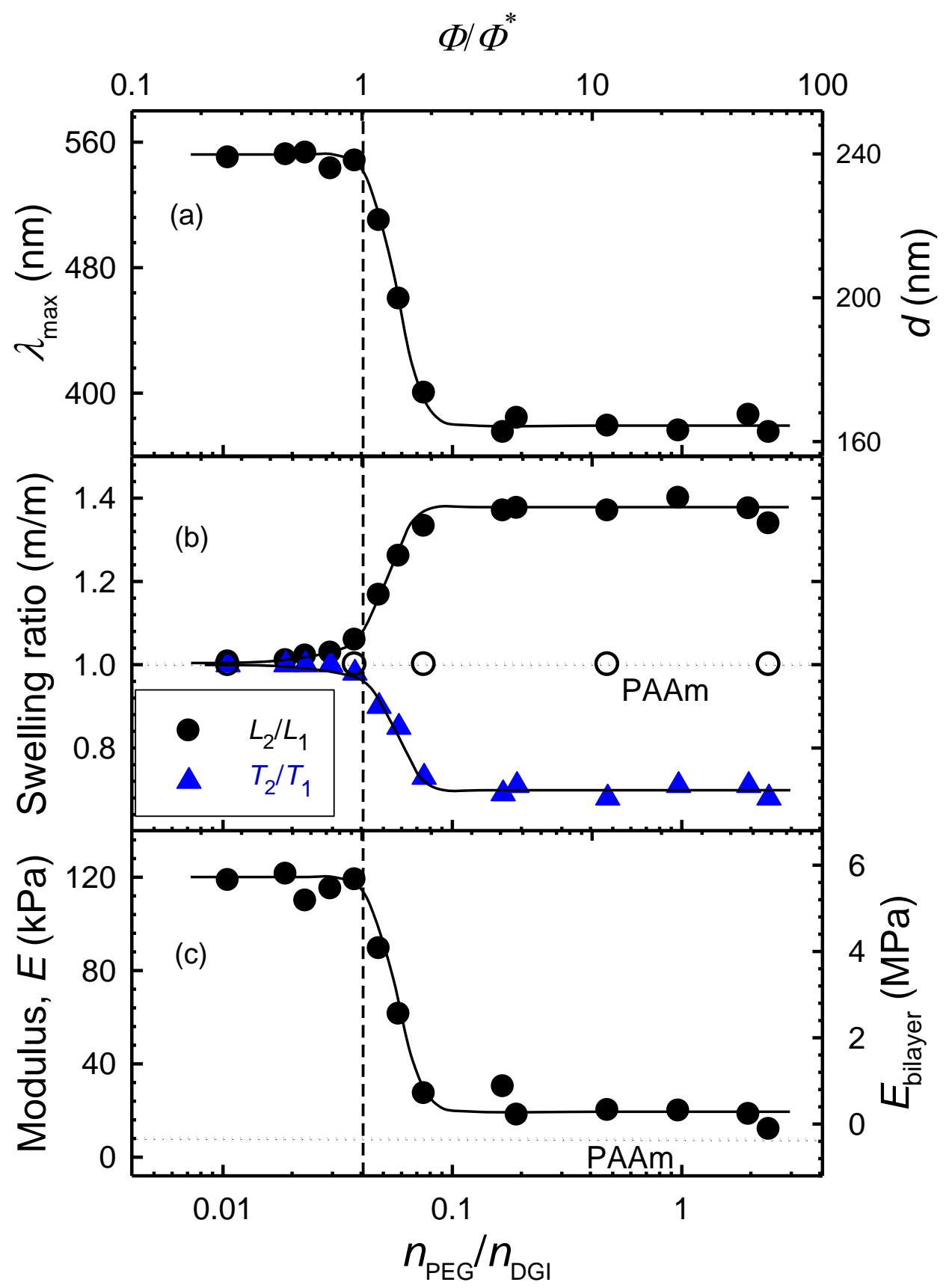

Figure 3: $\lambda_{\max }$ (a), swelling ratio (b), and modulus (c) are plotted against the PEG concentration. All the parameters show a sharp transition at a PEG concentration where $n_{\mathrm{PEG}} / n_{\mathrm{DGI}} \sim 0.4$. In excellent agreement with the estimated overlap concentration of PEG chain $\left(\Phi / \Phi^{*}=1\right)$, the transition appears at $\Phi / \Phi^{*} \sim 1$. 

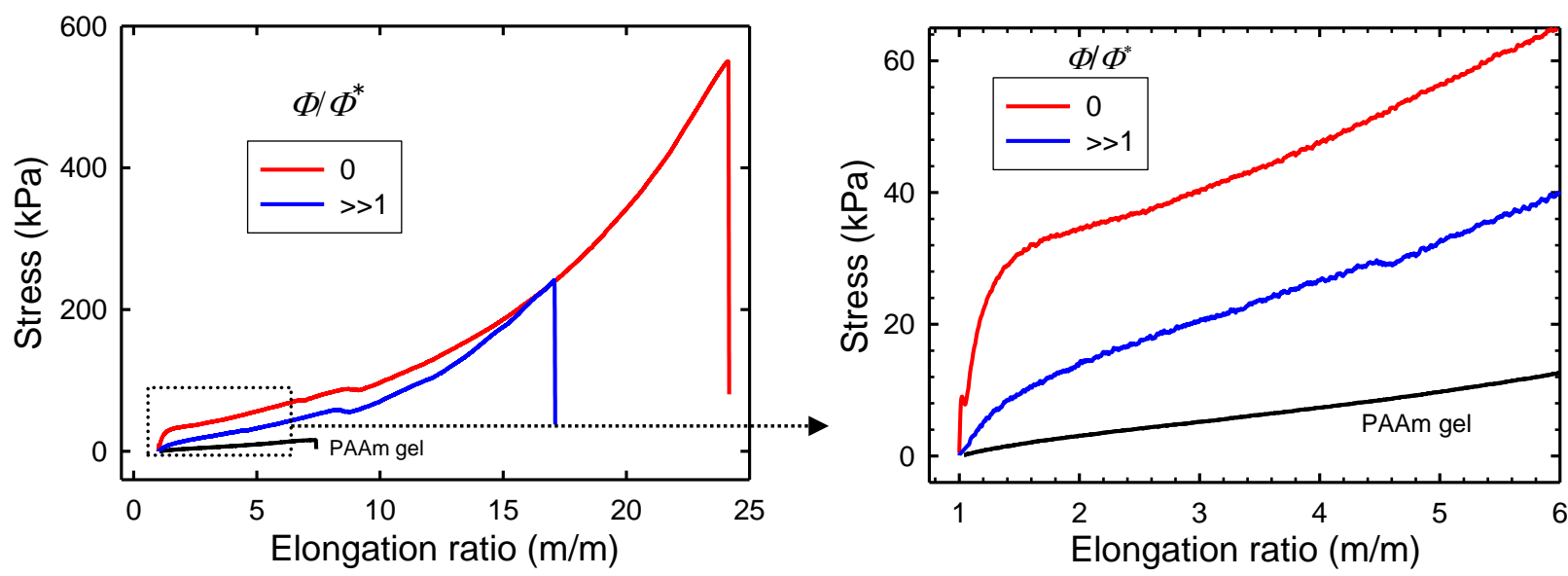

Figure 4: Stress-strain curves for the PAAm gel, PDGI/PAAm gel $\left(\Phi / \Phi^{*}=0\right)$, and the PDGI/PAAm gel swelled in high concentration of PEG $\left(\Phi / \Phi^{*}>>1\right)$. 
(a)

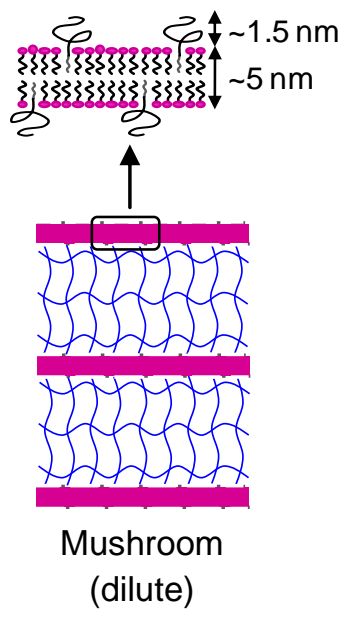

(b)

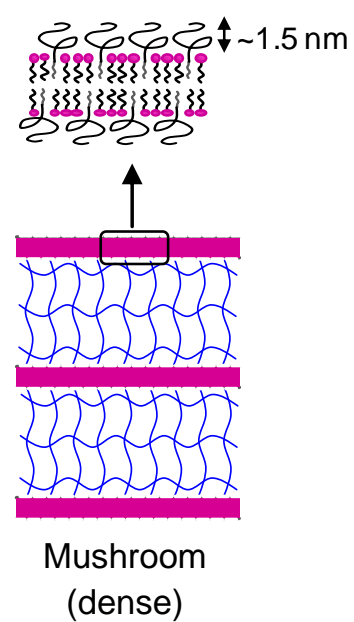

(c)

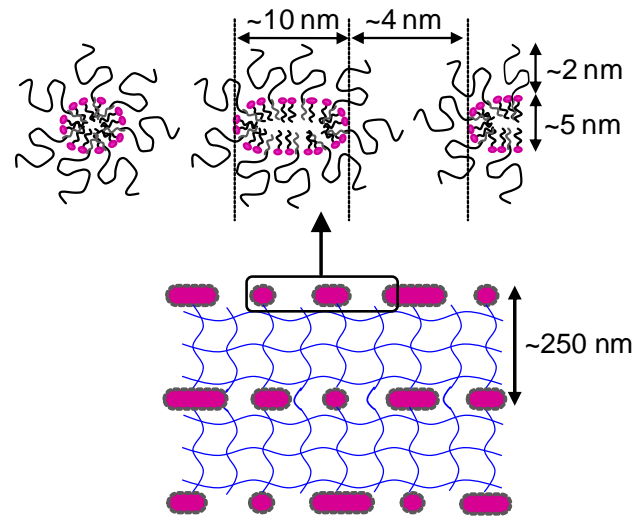

Brush

(bilayer-micelle)

Figure 5: Schematic illustration of the PEG grafting with different polymer chain conformations (a) mushroom (dilute; $\Phi / \Phi^{*}<<1$ ), (b) mushroom (dense; $\Phi / \Phi^{*} \sim 1$ ), and (c) brush $\left(\Phi / \Phi^{*}>>1\right)$. The polymer brush leads to a transition from bilayer phase to bilayermicelle phase which create many tunnels in the bilayer membrane as well as in the whole gel. 


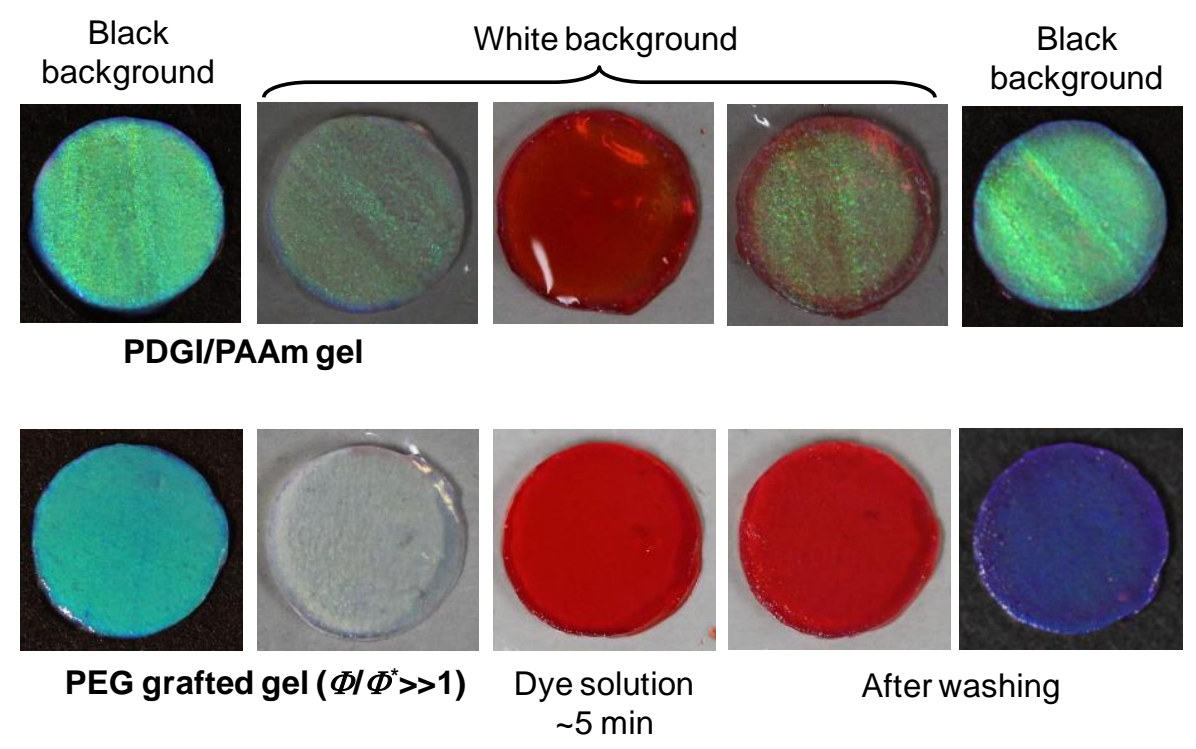

Figure 6: Diffusion of Allura red dye in both grafted ( $\left.\Phi / \Phi^{*}>>1\right)$ (upper row) and non-grafted (bottom row) PDGI/PAAm gel. After immersing both the gels in dye solution for 5 min (images in $3^{\text {rd }}$ column), both become red. After washing with water, the grafted gel still remains red in the whole sample, whereas the non-grafted gel show red only in the edge of the gel whereas the middle part are still same as that before dying (images in $4^{\text {th }}$ column). Comparing the images for grafted and non-grafted gels taken at black background, one can see easily that the grafted gel changes it color completely from green to blue but the nongrafted gel changes its color from green to blue only in the edge. This indicates the diffusion of dye through the membrane nano-channel in the grafted gel. 